\title{
Adaptive Difficulty with Dynamic Range of Motion Adjustments in Exergames for Parkinson's Disease Patients
}

\author{
Sandra Siegel and Jan Smeddinck \\ TZI Digital Media Lab, University of Bremen \\ Bibliothekstraße 1, 28359 Bremen, Germany \\ \{siegel, smeddinck\}@tzi.de
}

\begin{abstract}
Motion-based video games offer great promise in the support of traditional physiotherapy and are currently explored in a growing number of research projects. With a focus on Parkinson's disease (PD) patients, their therapeutic needs and strong individual differences in capabilities, this work describes an approach to dynamic difficulty adjustments in an exergame tailored for PD patients. The automated ongoing adjustment of the required range of motion, the amplitude parameter, is introduced as an important aspect of such adaptations. Results from a first case study suggest that the approach is viable and appreciated by therapists, yet could benefit from increased flexibility.
\end{abstract}

Keywords: serious games, parkinson's disease, motion-based games, exergames, difficulty adaptation, elderly entertainment.

\section{Introduction}

Parkinson's disease (PD) is characterized by a variety of motor and non-motor symptoms [1]. Regular physical activity in therapy sessions and auxiliary exercises at home play a central role in slowing down the progression of the neurological disease and in improving the patients' quality of life [2] It is important to motivate patients to participate in these activities and to put effort into performing the often repetitive exercises. Exergames, as motion-based video games, offer great promise in augmenting existing therapy sessions and home exercising by providing additional guidance, feedback and motivation. Our work is based on the WuppDi! exergames suite for PD patients (cf. [3]). Evaluations of this project have shown that the chance of enriching the spectrum of tools around physiotherapy is appreciated by patients and therapists alike.

However, the target group of PD patients has very specific requirements that are dominated by the need to cater for strong individual differences in the mental and physical abilities and limitations of the players that result from different states of disease and symptoms, effects of normal ageing and fluctuations over the course of a day [2. Typical game-difficulty modes (e.g. easy, medium, hard) encompass multiple game-play variables and do not allow for the required finegrained control. 
We introduce a system that implements an ongoing adaptation of difficulty parameter settings for individual players, focusing on the automated adaptation of their range of motion (ROM). The system, which also adapts parameters for speed and accuracy, was tested in an initial case study over the course of three weeks, in order to determine whether the individual settings can successfully be adjusted over time, aiming for an improved range and quality of motion, while providing a positive game experience.

\section{A Brief Overview of DDA for Exergames and a PD Specific Implementation}

Dynamic Difficulty Adjustments (DDA) are an important matter of research in relation to motion-based games for the support of physiotherapy for PD patients. The need for personalization in order to accommodate for strong differences in capabilities between individuals is a core requirement that has been identified in prior research [3] and related research with other heterogeneous target-groups, such as institutionalized older adults 4 and stroke patients [5] This adaptation needs to be automated, because therapists cannot afford to make frequent manual adjustments for every individual patient. To date, related research seldom focused on the specific use-case of exergames for PD and largely omitted the aspect of automatic ROM adaptations, which we discuss here as the game-difficulty parameter amplitude. In addition to the more commonly studied difficulty parameters for speed and accuracy [56], the amplitude is an important aspect, since wide fluent movements are a crucial component of PD physiotherapy [3].

We implemented and tested DDA for Sterntaler, one of the WuppDi! games [3. In the game, the player is tracked by a Kinect 1 sensor and follows differently shaped trails of stars with one hand. The position of the playing hand is mapped to a hand-shaped cursor that is used to collect the stars for points. Here, game difficulty is composed of the time available to collect all stars before they automatically disappear (speed), the size of the cursor with which the player has to hit the stars (accuracy) and the amplitude of movements the player has to perform to reach all stars. The latter is controlled by a scale factor that is applied to the mapping of tracked movements to the cursor, making it easier or harder to reach stars further away from the player.

The three difficulty parameters are automatically adapted after each game round based on the player's performance in that round and additionally slightly decreased for the first game of a new game session in order to provide for a warmup round. Speed and accuracy settings change based on the rate of collected to non-collected stars and the completion time. For the adaptation of the amplitude setting, the collection rate is weighted by the distance of stars from the rest position of the play hand. Additionally, the motion profile of the play hand is consulted for inferring if non-collected stars were not accessible or if they could have been reached by the player (i.e. the player has moved his hand to the

${ }^{1}$ http://wWw.kinectforwindows.org 
respective position before). Optionally, a calibration based on three predefined poses can be performed to determine a suitable amplitude setting for the first game and to constrain dynamic amplitude adaptation to a reasonable maximum. This process must be monitored by therapists and the results can be manually adjusted to match therapeutic constraints or goals.

\section{Case Study}

The system was applied in a case study with three male PD patients aged 64, 76 , and 76, spanning five sessions per participant over a period of three weeks. Each participant played 16 game rounds with either hand. The evaluation was based on observational data, self-reported ratings of participants concerning the perceived difficulty and their game experience, game logs (difficulty settings, performance data and motion profiles) and video recordings that were analyzed together with associated physiotherapists. Due to the limited scope of this paper we focus on presenting the results related to amplitude adaptation (cf. [7]).

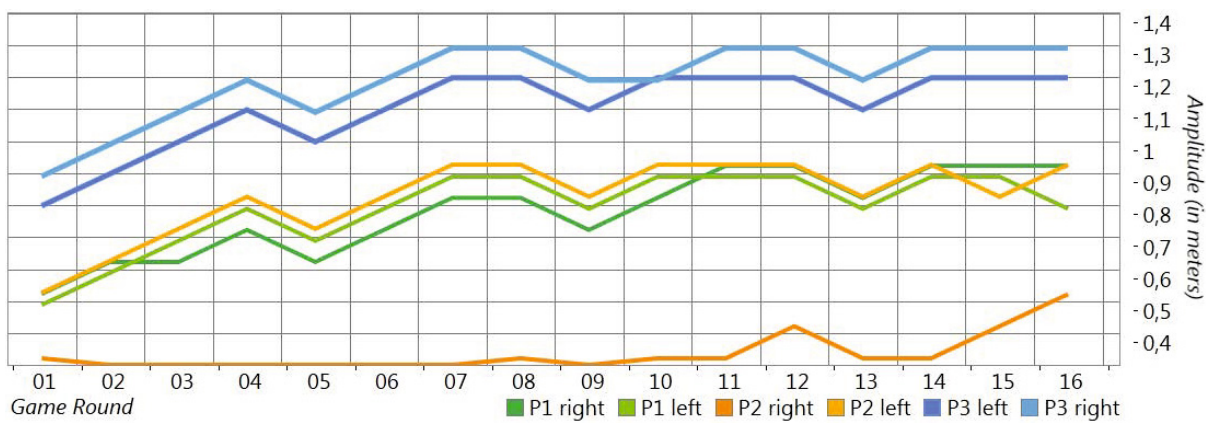

Fig. 1. The amplitude settings, which correspond to the diameter of movements necessary for covering the screen, for the three participants over all game rounds

The course of the amplitude settings is depicted in figure [1] The starting settings applied in the first round were determined by calibration and settled $40 \mathrm{~cm}$ below the calibrated amplitude. These settings are quite distinctive as they respond to the different motion capabilities of the three participants and their body height (ca. 1,7m for P1/P2 and 1.98m for P3). As expected, due to the easy start settings, the required ROM steadily increases in almost all cases during the first game rounds until the calibrated constraints are reached. P2 (right) differs from the general trend due to the participant's individual problems in raising his right arm vertically and reaching the central upper area of the screen. Physiotherapists, when analyzing video recordings at different amplitude levels, underlined the importance of the provided individual and dynamic amplitude settings. The high level settings were seen as increasingly suitable and effective as participants needed to fully stretch their arms and their back. 
While the upward trend in amplitude settings clearly illustrates the adaptation mechanism at work and therapists confirm that the adaptation approach generally provided suitable amplitude settings for our participants, some issues remain that demand for more flexibility in the adaptation mechanics: For P2 raising his right arm vertically was difficult, while stretching it to the sides was well possible, resulting in a low amplitude setting that did not get him to exploit his whole horizontal ROM. P1 suffers from a severely flexed spine (camptocormia) and an important focus in his exercises lies on stretching his trunk. Therapists therefore recommend to select star trails that motivate vertical movements and require a large range of motion while restricting the range horizontal movements, since these could possibly compromise his balance.

\section{Conclusion}

DDA in exergames have the potential to make them more useful and efficient, especially for heterogeneous target-groups with shifting needs like PD patients. The case study illustrated how such adaptations can work based on heuristic performance measures and highlighted the importance of an amplitude parameter in exergames for PD patients. However, the data analysis and therapist comments suggest that the system needs to be more flexible and allow for finegrained control to encompass individual strengths and weaknesses (e.g. vertical vs. horizontal ROM). A more distinctive area-based performance analysis and more frequent live adaptations, based on a wider spectrum of feedback metrics, could be viable approaches to these challenges. Lastly, improving and evaluating DDA in exergames requires long-term studies with more subjects and the possibility to correlate game-performance data to reliable medical data.

\section{References}

1. Goldenberg, M.: Medical management of parkinson's disease. P\&T 33(10) (2008)

2. Parkinson's Disease Society: The Professional's Guide to PD, London (2007)

3. Assad, O., Hermann, R., Lilla, D., Mellies, B., Meyer, R., Shevach, L., Siegel, S., Springer, M., Tiemkeo, S., Voges, J., Wieferich, J., Herrlich, M., Krause, M., Malaka, R.: Motion-Based Games for Parkinson's Disease Patients. In: Anacleto, J.C., Fels, S., Graham, N., Kapralos, B., Saif El-Nasr, M., Stanley, K. (eds.) ICEC 2011. LNCS, vol. 6972, pp. 47-58. Springer, Heidelberg (2011)

4. Gerling, K.M., Livingston, I.J., Nacke, L.E., Mandryk, R.L.: Full-Body MotionBased game interaction for older adults. In: CHI 2012: Proceedings of the 30th International Conference on Human Factors in Computing Systems (2012)

5. Alankus, G., Lazar, A., May, M., Kelleher, C.: Towards customizable games for stroke rehabilitation. In: Proceedings of the 28th International Conference on Human Factors in Computing Systems, pp. 2113-2122 (2010)

6. Geurts, L., Vanden Abeele, V., Husson, J., et al.: Digital games for physical therapy: fulfilling the need for calibration and adaptation. In: Proceedings of the Fifth International Conference on Tangible, Embedded, and Embodied Interaction, TEI 2011, pp. 117-124. ACM, New York (2011)

7. Siegel, S.: Adaptive Difficulty in Exergames for Parkinsons Disease Patients. Unpublished master's thesis. University of Bremen, Bremen (2012) 